\title{
Buried Object Discrimination in a Ground Penetrating Radar Radargram
}

\author{
M.P. Priyadarshini and Dr.G. Indumathi
}

\begin{abstract}
Ground Penetrating Radar (GPR) is a nondestructive technique used for the location of objects or interfaces buried beneath the earth's surface or located within a visually opaque structure. This research work proposes techniques for buried object discrimination for the images generated by GPR by using GPR frequency-domain spectral features. The motivation for this approach comes from the fact that landmine targets and clutter objects often have different shapes and/or composition, yielding different Energy Density Spectrum (EDS) that may be exploited for their discrimination. The proposed system comprises cascade of two stages: Pre - Processing Stage, followed by Discrimination Stage. Pre - Processing eliminates the ground bounce and clutter to get an enhanced image. Enhancement is followed by Landmine or clutter Discrimination using Energy Density Spectrum. All implementations are done using MATLAB.
\end{abstract}

Keywords--- Auto - Correlation, Contrast, Energy Density Spectrum (EDS), Ensemble Average, Entropy, Fourier Transform, Radargram, Spectral Frequency

\section{INTRODUCTION}

G ROUND Penetrating Radar (GPR) is a non-destructive geophysical method that produces a continuous crosssectional profile or record of subsurface features, without drilling, probing, or digging. GPR profiles are used for evaluating the location and depth of buried objects and to investigate the presence and continuity of natural subsurface conditions and features. GPR can be used in a variety of media, including rock, soil, ice, fresh water, pavements and structures. It can detect objects, changes in material, and voids and cracks [1], [2].

GPR generated images called Radargram (GPR Raw Image) is said to have three main signal components: target (object) component, ground reflected signal component and clutters [1]. Therefore, processing of the GPR raw image is must. Figure. 1 shows the flow of the proposed techniques. It involves two stages: Pre -Processing Stage, followed by Discrimination. Pre - Processing eliminates the ground bounce and background clutter to get an enhanced image. Enhancement is followed by Landmine or clutter Discrimination using Energy Density Spectrum (EDS).

M.P. Priyadarshini Junior Research Fellow, VGST DST - GOK, C.M.R. Institute of Technology, Bangalore, India. E-mail: priyadarshini.mp@gmail.com

Dr.G. Indumathi, Prof. \& HOD, C.M.R. Institute of Technology, Bangalore, India.E-mail: indu_cmrit@rediffmail.com

DOI: 10.9756/BIJAIP.10188

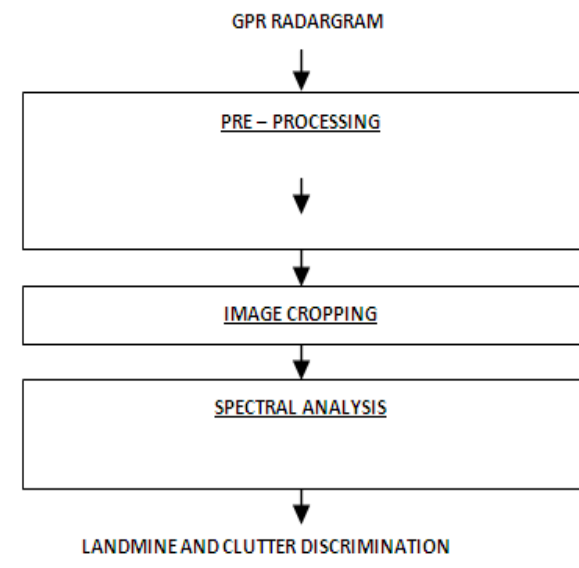

Figure 1: Flow of Proposed Techniques for Target Detection

\section{PRE- PROCESSING}

Before the information in the raw radargram can be utilized correctly, it must first be processed to remove undesired system and ground effects. This involves background clutter removal, path loss compensation, antennaseparation rectification and low-pass filtering [5].

Main aim of GPR Pre - Processing is to remove the ground bounce and background clutter and to get a uniform background which leads to enhanced target signal. This is done in two stages: Ensemble Averaging [5] and Entropy Optimized Contrast Stretching [6].

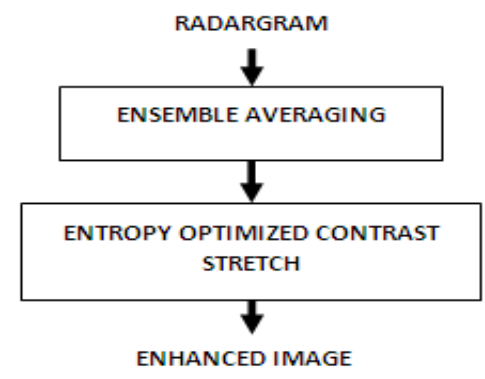

Figure 2: Pre - Processing Stages

\section{A. Ensemble Averaging}

For a downward looking GPR system, which has the geometry that transfers the most radiated power to the subsurface, the collected data is severely contaminated by specular reflections from the ground surface. These reflections, usually referred to as the ground-bounce, can greatly surpass and hide the weak return of a shallowly buried landmines. The ground-bounce effect can be eliminated by positioning the antennas in direct contact with the soil so that no ground-bounce is allowed to form. For obvious reasons, this is not a feasible solution for the buried object detection application of GPR. To be able to detect a possible buried landmine in a reliable manner, we instead have to estimate and remove the ground bounce from the data without impairing the return of the mine significantly [1], [2]. 
Ground bounce removal is achieved by subtracting from each scan an ensemble average of the stack of A-scans over the region of interest [5]. Here it is assumed that the material properties vary randomly about a location independent mean, and that the target indications are present in a relatively small number of measurements. The image is divided into vertical strips of approximately 60 scans (or the equivalent of three metres), and then the ensemble mean of each row of pixel intensities across each strip is subtracted. Using the same notations as $-s(i)$ : the unprocessed A-scan and $w_{\text {in }}$ : the width of the strip of scans starting from scan number $j_{\mathrm{bg}}$, the processed A-scan $-\overline{s(l)}$ is calculated using equation (1)

$$
\overline{s(l)}=s(i)-\frac{1}{W_{i n}} \sum_{j=j_{b g}}^{j_{b g}+W i n-1} S(j)
$$

Ground bounce associated with a particular region may be removed by specifying the corresponding values of $j_{\mathrm{bg}}$ and $w_{i n}$. This technique is particularly well-suited for instances where the targets are well-separated.

\section{B. Entropy Optimized Contrast Stretching}

The contrast of the landmine and surroundings is still poor due to their similar electromagnetic properties as well as other clutter contributions [6].

Background clutter represents small unwanted reflections caused mainly rocks, antenna coupling, buried metals etc.

\section{- $\quad$ Contrast Stretching (CS)}

Frequently, an image is scanned in such a way that the resulting brightness values do not make full use of the available dynamic range. By stretching the histogram over the available dynamic range we attempt to correct this situation. The appropriate transformation is given by equation (2)

$v=\left\{\begin{array}{l}\alpha u, 0 \leq u \leq a \\ \beta(u-a)+v_{a}, a \leq u \leq b \\ \gamma(u-b)+v_{b}, b \leq u \leq L\end{array}\right.$

Here $a=$ lower boundary and $b=$ upper boundary of the gray levels in the histogram. " $u$ " is the pixel intensity. $\alpha, \beta$ and $\gamma$ are the constant CS parameters which varies with the image.

The percentage linear contrast stretch uses a specified minimum and maximum value that lie in a certain percentage of pixels from the mean of the histogram. Applying the contrast stretch in GPR image will enhance the contrast of the object reflected signals and make them easy to detection. Consider an image with a minimum brightness value of 0 and a maximum value of 255. Assume we choose a lower boundary of 10 and upper boundary of 200 in the contrast stretch, the pixels in the original image with a pixel value less than 10 or greater than 200 are changed to 0 and 255 , respectively. And the image is linearly stretched between 0 and 255. Therefore, part of the original image that is of lowcontrast now is of higher contrast. This is shown in the figure3.

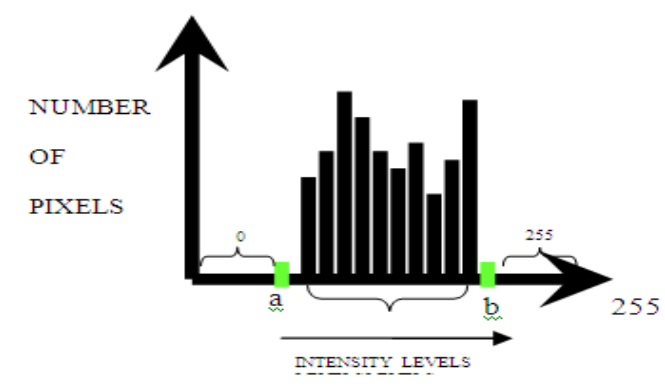

Figure 3: Image Histogram with Boundaries a and $b$

Hence the Contrast Stretch can be now expressed as follows:

$$
v=\left\{\begin{array}{l}
0,0 \leq u \leq a \\
\beta(u-a)+v_{a}, a \leq u \leq b \\
255, b \leq u \leq 255
\end{array}\right.
$$

\section{- Limitations of Contrast Stretching}

The difficulty in the percentage linear contrast stretch is to determine the lower and upper percentage boundaries. Boundaries that are too far apart will have little effect on the GPR images while boundaries too close will in adversely enhance a lot of details of the GPR images while most of them are noise and clutters. Therefore it is important to have a criterion in choosing the proper boundaries. We can write the $\mathrm{CS}$ as an operator such that -

$$
Y=C S(X, a, b)
$$

where $Y$ is the resulting image of size $M \times N$ and $a$ and $b$ are the lower and upper boundary, respectively, and $0<\mathrm{a}<\mathrm{b}$ $<255$.

\section{- Entropy Optimized Contrast Stretching}

For the purpose of detection, we look for the CS that decomposes a GPR image into three pans, the specular reflection, the object reflected signals and possibly a homogeneous background. A measurement of the homogeneity of an image is its entropy. Entropy measures amount of information carried in an image. Large entropy corresponds to a large amount of information and vice versa. Intuitively, a homogeneous image has smaller entropy than an inhomogeneous image.

We use approximation to the entropy, namely, the varimax norm. The varimax norm of an image is defined as -

$$
\Omega(X)=\frac{\left[\sum_{m}^{M} \sum_{n}^{N} X^{2}(m, n)\right]^{2}}{\left[\sum_{m}^{M} \sum_{n}^{N} X^{4}(m, n)\right]}
$$

An image of small entropy has a small varimax norm and vice versa. An identically homogeneous image, i.e., a constant image, has entropy of one, which is the smallest varimax norm. Any inhomogeneous image will have a varimax norm larger than one. In entropy optimized CS, we start with a small lower boundary $a$ and a large upper boundary $b$. Then we increase $a$ and decrease at the same rate and calculate the varimax norm of the resulting image. When a minimum is reached, it is believed that at least a sub-optimal set of boundaries has been encountered and the above process stops. To find the optimal boundaries corresponding to the minimum entropy, we need to find the point $k$ such that varimax norm is minimal [4]. 


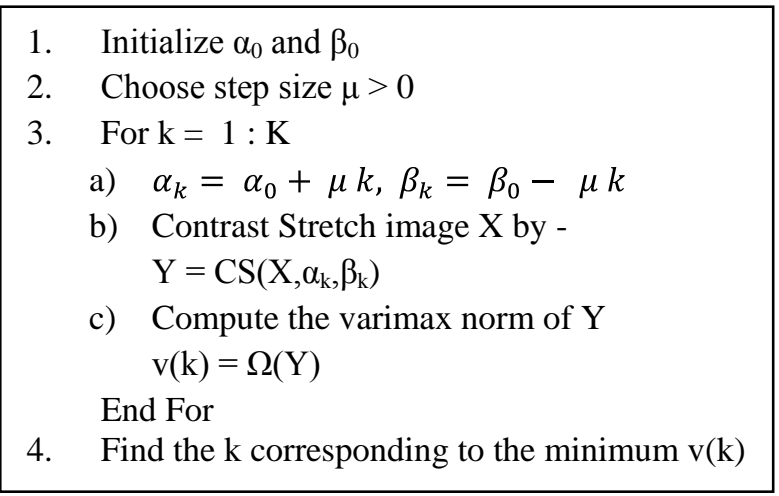

Figure 4: Entropy Optimized Contrast Stretching

We start with a lower boundary $\alpha_{0}=0.01$ and upper boundary $\beta_{0}=0.99$ and a step size $\mu=0.01$. the lower boundary increases as -

$\alpha_{k}=\alpha_{0}+\mu k$

Where $\mathrm{k}=1,2, \ldots \ldots, 20$.

Similarly the upper boundary is decreased as -

$\beta_{k}=\beta_{0}-\mu k$

As the boundaries close in, the object reflected signals, i.e. the hyperbolic curve in the image becomes clearer and details begin to come forward.

For data 1 and data 2 , the optimal value of $\mathrm{k}$ is 12 .

\section{DISCRIMINATION}

The GPR signal from a landmine is dependent on the mine's size, shape, and composition, as well as its burial depth and orientation. In addition to the properties of the mine itself, electrical characteristics of the soil also play an important role on the signature of landmines and clutter. For example, if the dielectric constant of the mine and soil are similar, the electrical discontinuity manifested by the mine-soil heterogeneity may be small, yielding a weak landmine signature. To address this problem, one may lower the detection threshold, thereby increasing the probability of detecting mines with weak signatures; however, this typically will cause a significant increase in the number of false alarms.

Rather than simply using the (often weak) signature amplitude to perform detection of landmines, one may consider exploiting the spectral properties of the signature to use potential mine-specific features. In this research work, discrimination based on spectral features extracted from the entire GPR waveform signature is performed. The rationale to exploit the spectral characteristics for classification is that landmine targets and clutter objects often have different shapes as well as composition, which yields different amounts of energy return at different frequencies, and hence different energy density spectra.

Signals which have finite energy possess Fourier transform and they are represented in their spectral domain by their Energy Density Spectrum. The distribution of energy with frequency is called Energy Density Spectrum [11].

\section{- Estimating Energy Density Spectrum}

First, the auto-correlation is computed from the GPR radargram and then the Fourier transform of the autocorrelation is computed. Since it is well known that the spectral density function of a signal is given by the signal Fourier transform of auto-correlation.

The clutter objects all have a strong GPR energy return. Interestingly though, their spectra have shapes quite different from those of the landmines. As a result, it is expected that the EDS will be useful in increasing the detection of some weakscattering plastic landmines, and at the same time providing discrimination ability between a landmine and clutter objects. It should be noted that there is a limit to which the EDS can be provided in the discrimination between landmine and clutter objects.

Landmines have a sharp peak at a single frequency in their Energy Density Spectrum, while the Clutters have irregular Energy Density Spectrum [11].

\section{RESULTS}

\section{- DATA 1}

The fig. 4, shows the radargram generated by the GPR, which is corrupted with Ground Bounce (GB) and clutters. This radargram was subjected to the proposed techniques.

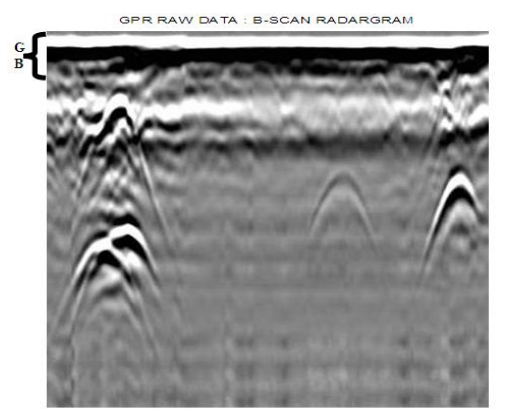

Figure 4: GPR Radargram (RAW DATA)

A. Pre-Processing

a) Step 1: Ensemble Averaging: for Ground Bounce (GB) removal

Ensemble Averaging was applied on the fig.4 to remove the ground bounce. The result of Ensemble Averaging is shown in figure 5 .

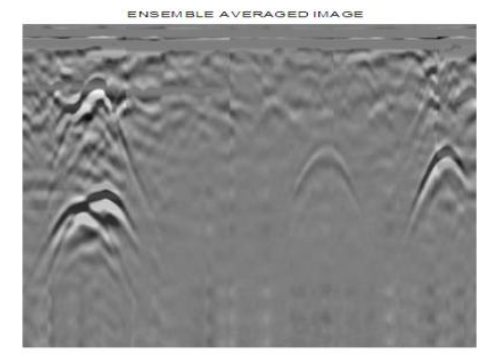

Figure 5: Ensemble Averaged Image

\section{b) Step 2 : Entropy Optimized Contrast Stretch : for Background Clutter Removal}

Entropy Optimized Contrast Stretching was applied on the figure 5 to remove the background clutter. The result of Entropy Optimized Contrast Stretching is shown in figure 6. The hyperbolic signatures of the buried objects are enhanced. 


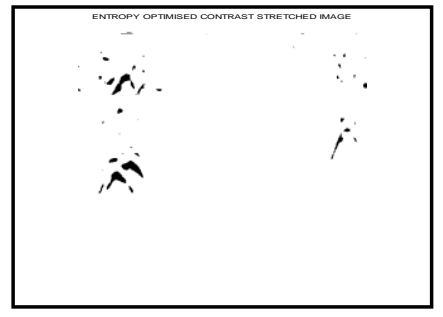

Figure 6: Entropy Optimized Contrast Stretched Image

\section{B. Image Cropping and Energy Density Spectrum Estimation}

Figure 6 reveals four hyperbolas. Therefore there are four buried objects. Each hyperbola is cropped and its Energy Density Spectrum is estimated and analysed.
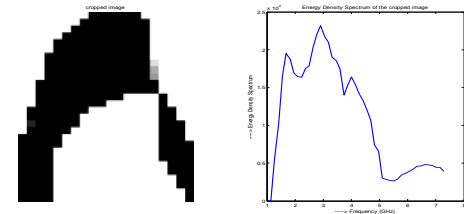

Figure 7: Hyperbola 1(left) and its Energy Density Spectrum (Right)

Energy Density Spectrum of the cropped hyperbola 1 has sharp peak in more than one frequency, i.e. it has a irregular Energy Density Spectrum, hence it can be concluded to be a clutter.
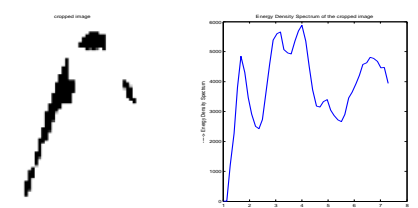

Figure 8: Hyperbola 2(left) and its Energy Density Spectrum (Right)

Energy Density Spectrum of the cropped hyperbola 2 has sharp peak in more than one frequency, i.e. it has a irregular Energy Density Spectrum, hence it can be concluded to be a clutter.
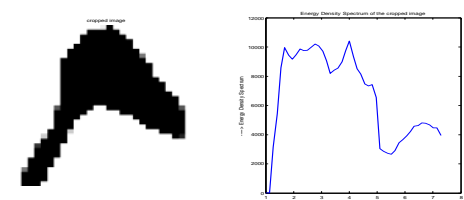

Figure 9: Hyperbola 3(left) and its Energy Density Spectrum (Right)

Energy Density Spectrum of the cropped hyperbola 3 has sharp peak in more than one frequency, i.e. it has a irregular Energy Density Spectrum, hence it can be concluded to be a clutter.

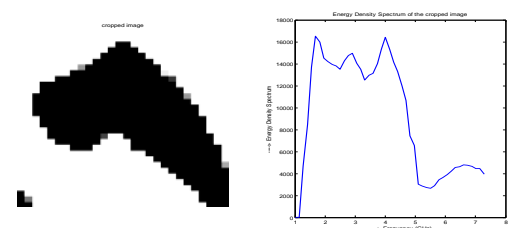

Figure 10: Hyperbola 4(left) and its Energy Density Spectrum (Right)

Energy Density Spectrum of the cropped hyperbola 4 has sharp peak in more than one frequency, i.e. it has a irregular Energy Density Spectrum, hence it can be concluded to be a clutter.

\section{- DATA 2}

The figure 11, shows the radargram generated by the GPR, which is corrupted with ground bounce (GB) and clutters. This radargram was subjected to the proposed techniques.

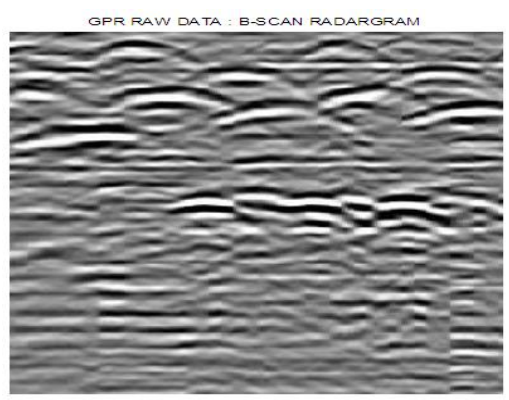

Figure 11: GPR Radargram (RAW DATA)

\section{A. Pre-Processing}

a) Step 1: Ensemble Averaging: for Ground Bounce (GB) Removal

Ensemble Averaging was applied on the fig. 11 to remove the ground bounce. The result of Ensemble Averaging is shown in figure 12.

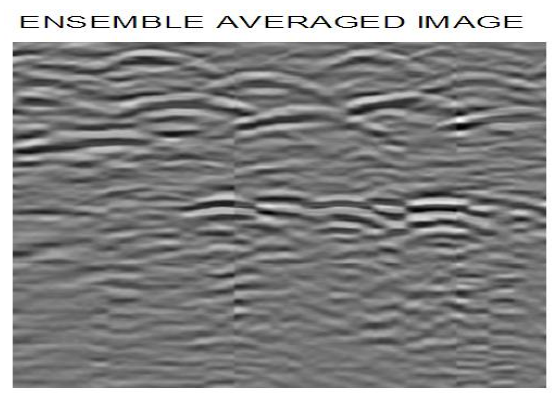

Figure 12: Ensemble Averaged Image

b) Step 2 : Entropy Optimized Contrast Stretch : for Background Clutter Removal

Entropy Optimized Contrast Stretching was applied on the figure 12 to remove the background clutter. The result of Entropy Optimized Contrast Stretching is shown in fig.13. The hyperbolic signatures of the buried objects are enhanced.

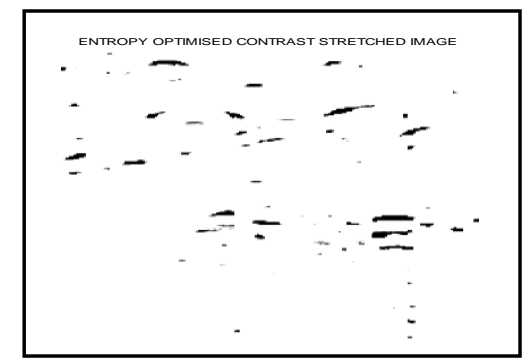

Figure 13: Entropy Optimized Contrast Stretched Image

B. Image Cropping and Energy Density Spectrum Estimation

Figure 13 reveals four hyperbolas. Therefore there are four buried objects. Each hyperbola is cropped and its Energy Density Spectrum is estimated and analysed. 

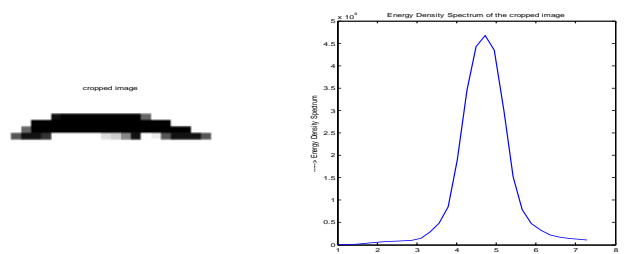

Figure 14: Hyperbola 1(left) and its Energy Density Spectrum (Right)

Energy Density Spectrum of the cropped hyperbola 1 has sharp peak at one frequency; hence it can be concluded to be a landmine.

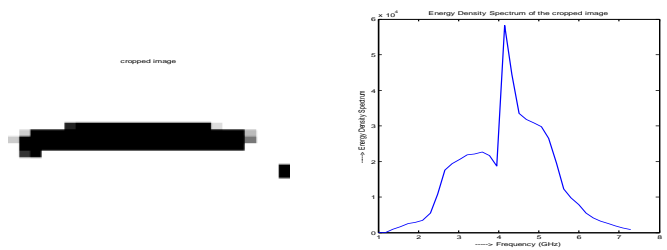

Figure 15: Hyperbola 2(left) and its Energy Density Spectrum (Right)

Energy Density Spectrum of the cropped hyperbola 2 has sharp peak at one frequency, hence it can be concluded to be a landmine.
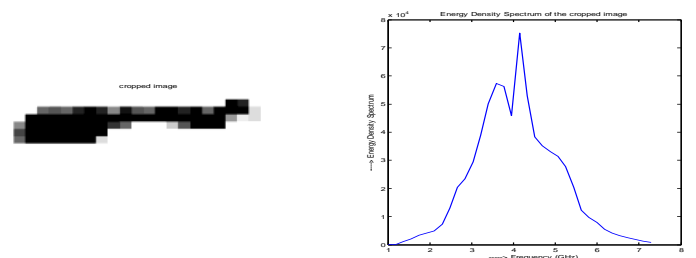

Figure 16: Hyperbola 3(left) and its Energy Density Spectrum (Right)

Energy Density Spectrum of the cropped hyperbola 3 has sharp peak at one frequency; hence it can be concluded to be a landmine.

Energy Density Spectrum of the cropped hyperbola 4 has sharp peak in more than one frequency, i.e. it has a irregular Energy Density Spectrum, hence it can be concluded to be a clutter.
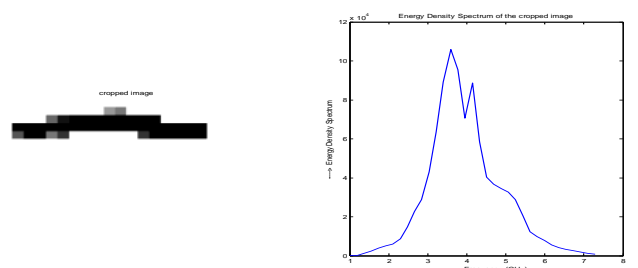

Figure 17: Hyperbola 4(left) and its Energy Density Spectrum (Right)

\section{CONCLUSION AND FUTURE ENHANCEMENT}

This work investigated the spectral characteristics of a target obtained by GPR measurements to improve landmine detection and clutter discrimination. Ensemble Averaging and Entropy Optimized Contrast Stretching proved to be good for the ground bounce and background clutter removal and to enhance low contrast landmines. Using EDS it was possible to discriminate the clutter objects that had different size, geometry and composition with a landmine target. In practice, there may be some clutter objects that have similar geometry and composition characteristics as a landmine. As a result, the proposed technique will be more appropriate to be used as features that will be fused with other algorithm outputs

\section{ACKNOWLEDGMENT}

The authors would like to acknowledge the infrastructure support provided VGST - GOK. And also the support provided by the Board of Governors and Principal of C.M.R.I.T.

\section{REFERENCES}

[1] Harry. H. Jol, E "GPR : Theory and Applications. First edition,2009.

[2] David .J Daniels, "Ground Penetrating Radar", $2^{\text {nd }}$ Edition. Published by: The Institution of Electrical Engineers, London, United Kingdom, 2004.

[3] Rafael. C Gonzalez and Richards. E. Woods, "Digital Image Processing". $2^{\text {nd }}$ Edition, published by Prentice Hall Inc.,2002.

[4] Omer Dermirkay,Mushakam, Asyali and Prasanna L Sahu, "Image Processing with MATLAB : Application in Medicine and Biology". Published by CRC Press.

[5] W. Al - Nuaimy, Y. Huang, M. Nakhksh, M.T.C. Fang, V.T. Nguyen, and A. Eriksen, "Automatic Detection of Buried Utilities and solid objects with GPR using neural networks and pattern recognition", Journal of Applied Geophysics 43, 157-165, 2000.

[6] Xiaoyin Xu and Eric L. Miller, "Entropy Optimized Contrast Stretch to Enhance Remote Sensing Imagery," ICPR, 16th International Conference on Pattern Recognition (ICPR'02) - Vol. 3, Pp.30915, 2002.

[7] R.H. Stolt, "Migration by Fourier Transform", Geophysics, Vol. 43, No. 1, Pp, 23-48, Feb 1978.

[8] C. Stolt and Klaus - Peter,N,"Eccentricity Migration : A method to improve the imaging of pipes in RADAR reflector data", Proc. Of the fifth Int. Conf. on Ground Penetrating Radar.

[9] Enes Yigit, Serket Demirci and Caner Ozdemir, "Ground Penetrating Radar Image focussing using Frequency - Wavenumber based Synthetic Aperture Radar Technique", IEEE Trans. 2007.

[10] N. Otsu, "A Threshold Selection Method from Gray Level Histogram," IEEE Transaction on Systems, Man, and Cybernetics, Vol. SMC-9, No. 1, Pp. 62-66, 1978.

[11] K.C. Ho and Paul D. Gader, "An investigation of using the spectral characteristics from Ground Penetrating Radar for Landmine/Clutter discrimination ",IEEE transactions on Geoscience and Remote Sensing ,Vol.46, No.4, 1177-1191, 2008 